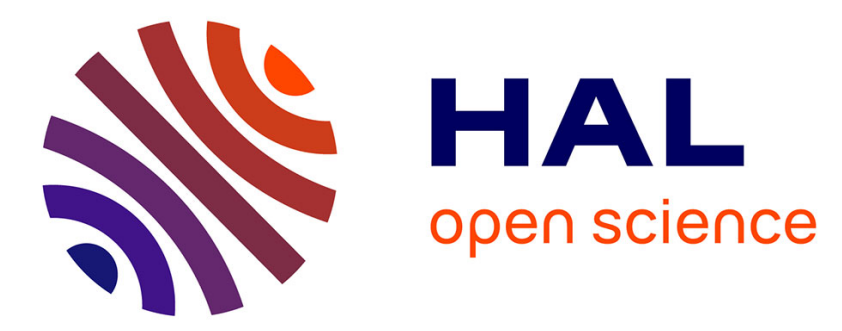

\title{
Regulation of the Hedgehog Morphogene Gradient
}

Gisela D'angelo, F Wendler, K Ayers, P P Thérond

\section{To cite this version:}

Gisela D'angelo, F Wendler, K Ayers, P P Thérond. Regulation of the Hedgehog Morphogene Gradient. Hedegehog signaling activation in human cancer and its clinical implications, pp.17 - 32, 2011, 10.1007/978-1-4419-8435-7_2. hal-03421575

\section{HAL Id: hal-03421575 \\ https://hal.science/hal-03421575}

Submitted on 15 Dec 2021

HAL is a multi-disciplinary open access archive for the deposit and dissemination of scientific research documents, whether they are published or not. The documents may come from teaching and research institutions in France or abroad, or from public or private research centers.
L'archive ouverte pluridisciplinaire HAL, est destinée au dépôt et à la diffusion de documents scientifiques de niveau recherche, publiés ou non, émanant des établissements d'enseignement et de recherche français ou étrangers, des laboratoires publics ou privés. 
See discussions, stats, and author profiles for this publication at: https://www.researchgate.net/publication/226683630

\section{Regulation of the Hedgehog Morphogene Gradient}

Chapter · April 2011

DOl: 10.1007/978-1-4419-8435-7_2

\section{CITATION}

1

4 authors, including:

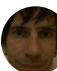

Franz Wendler

University of Sussex

39 PUBLICATIONS 2,249 CITATIONS

SEE PROFILE

Some of the authors of this publication are also working on these related projects:

Project Glioblastoma and its microenvironment. View project

Project LMTK3 confers chemo-resistance in breast cancer View project

\section{READS}

25

Katie louise Ayers

Murdoch Children's Research Institute

44 PUBLICATIONS 1,179 CITATIONS

SEE PROFILE 


\title{
Chapter 2 \\ Regulation of the Hedgehog Morphogene Gradient*
}

\author{
G. D’Angelo, F. Wendler, K. Ayers, and P. P. Thérond
}

Keywords Hedgehog - Secretion - Trafficking - Morphogenetic gradient

- Extracellular matrix • Heparan sulfate proteoglycans

\section{Introduction}

The development of a multicellular organism is controlled by a genetic program that manifests itself in proliferation, cell differentiation, and apoptosis, leading to the formation of functional organs. A small number of secreted molecules work as "instructors" during these processes [1, 2]. Among them are the Hedgehog $(\mathrm{Hh})$ family of proteins, which act from their source of production at short and long range. They trigger cell fate decisions by inducing a signaling cascade in the ligandreceiving tissues of invertebrates and vertebrates. Dramatic developmental abnormalities are observed in human embryos with compromised Hh signaling, and while great effort is being made to understand and manipulate the signaling cascade downstream of $\mathrm{Hh}$ receptor activation, less attention has been payed to the secretion and release of the Hh ligand itself. In this review, we will discuss recent progresses in the understanding of Hh ligand packaging and dispatch from producing cells and its consequences for gradient formation.

\footnotetext{
* The first two authors contributed equally to this manuscript.

P.P. Thérond $(\bowtie)$

Institut Biologie du Développement \& Cancer - IBDC,

Université de Nice Sophia-Antipolis, UMR6543 CNRS, Centre de Biochimie,

Parc Valrose, 06108 Nice cedex 2, France

e-mail: therond@unice.fr
} 


\section{Atypical Biosynthesis of Active Hh Signaling Peptide}

An evolutionarily conserved feature of $\mathrm{Hh}$ family proteins resides in their hydrophobic lipid modifications. Immature Hh protein enters the secretory pathway where proteolytic autoprocessing and lipidation produce an $\mathrm{N}$-terminal active Hh peptide modified on the C-terminus by a cholesterol moiety, both on Drosophila $\mathrm{Hh}$ and on its vertebrate counterpart Sonic Hedgehog (Shh) [3] (Fig. 2.1). Moreover, active Hh is also modified by the attachment of a stable amide-linked palmitic acid at the opposing N-terminal end $[4,5]$. This acylation is catalyzed by the Skinny Hedgehog protein (Skn or Ski, also called Rasp, Central missing and Sightless) [5-8] that belongs to the family of membrane bound O-acyl-tranferases (MBOAT) [9]. The importance of these dual lipid modifications is underscored by the fact that removal of these modifications interferes with the biological activity of $\mathrm{Hh}$ in vivo. Notably, mutations that affect the human Shh autocatalytic processing are associated with holoprosencephaly [10].

\section{Routing of Hh to the Plasma Membrane}

The presence of lipid moieties has a significant effect on Hh solubility and is, therefore, bound to affect intracellular trafficking to the plasma membrane in producing cells. Strikingly, although Hh lipidation is essential for its activity, the exact intracellular compartments in which these modifications take place during Hh secretion are not known. Nevertheless, we do know that the dependence of correct Hh intracellular trafficking on the cholesterol moiety has been demonstrated, as a mutant form of Hh lacking the cholesterol adduct shows significantly altered subcellular distribution [11]. In addition, a hydroxyl-oxygen present within the cholesterol is responsible for the cleavage of $\mathrm{Hh}$ [3], and as both autoproteolysis and cholesterol modification are linked, they likely occur in the same compartment. Sensitivity of the uncleaved Shh precursor to digestion with the EndoH glycosidase suggests that the autoprocessing reaction occurs before the medial Golgi [10]. In addition, two mutant forms of Shh (point mutations) that present a temperature-dependent misfolding and retention in the ER do not undergo autoprocessing cleavage. It is thus likely that cleavage and autoprocessing occur in a post-ER pre-medial Golgi compartment, such as the cis-Golgi. This would also be consistent with the availability of cholesterol as a critical determinant for cleavage, as cholesterol is found at only low levels in ER membranes and at higher levels in Golgi membranes [12]. This, however, must be experimentally determined, membranes and cleavage might alternatively be required for a unique or specific $\mathrm{Hh}$ ER-exit route.

The site of the second Hh modification, palmitoylation, is also an open question, as mouse tagged-Skn protein is mainly localized to the ER in CHO and HeLa cells $[13,14]$, whereas Drosophila Ski was found in the Golgi apparatus in insect Schneider cells [15]. It is also not known whether palmitoylation is conditional to 

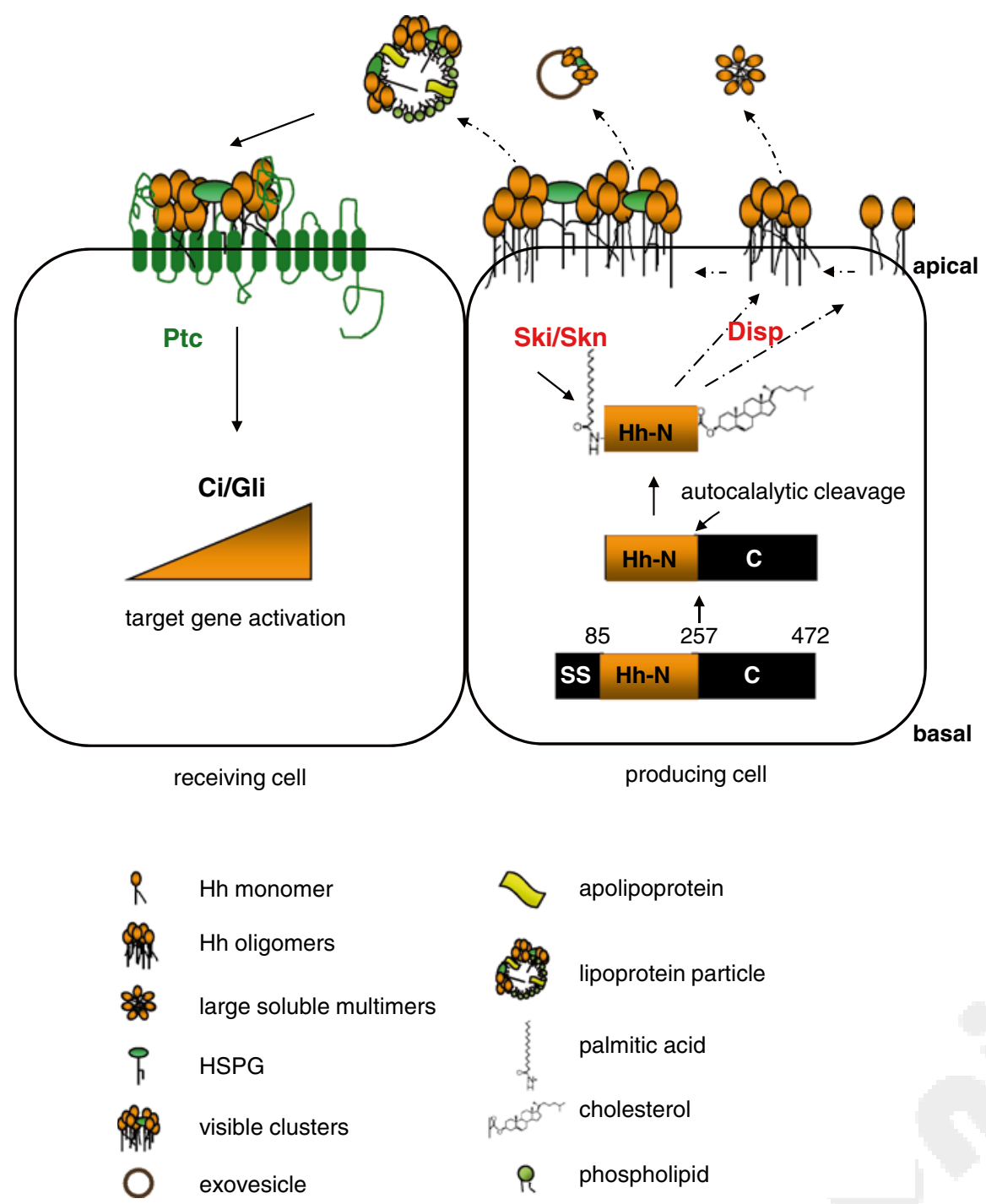

Fig. 2.1 A schematic diagram of Hh protein biogenesis, secretion, release, and transport to target cells. In Hh-producing cells, the immature Hh protein enters the secretory pathway where proteolytic autoprocessing and lipidation produce a $\mathrm{N}$-terminal active peptide modified on its $\mathrm{C}$-terminus by a cholesterol moiety. Additionally, a stable amide-linked palmitic acid is attached to its $\mathrm{N}$-terminus by the acyltransferase Ski/Skn. The active dually lipid-modified $\mathrm{Hh}$ is denoted as Hh-N. The destiny of the C-terminal fragment after cleavage is not known. The transmembrane protein Dispatched (Disp) promotes the secretion of monomers and/or oligomers of $\mathrm{Hh}-\mathrm{N}$ to the apical cell membrane. Monomers of Hh-N could self-associate spontaneously to form large soluble multimers and can be released in the extracellular lumen. Conversely, Hh-N oligomers at the cell surface could be selectively enriched in visible clusters along with heparan sulfate proteoglycans (HSPGs). Upon release from producing cells, the visible clusters may be transported across several cell diameters from the Hh-producing cells either into exovesicles and/or as an integral component of lipoprotein particles. The transport of $\mathrm{Hh}-\mathrm{N}$ by one or by the combination of the diverse carriers leads to transcriptional activation of $\mathrm{Hh}$ target genes (via the transcriptional activator $\mathrm{Ci} / \mathrm{Gli}$ ) in receiving cells upon its association with the transmembrane protein Patched (Ptc; Hh receptor). Activation of the Hh pathway can also be triggered by a membrane-associated Hh (not shown) 
cholesterol modification, as contradictory results have been offered. Analysis of recombinant human and rat Shh produced in a cell-based assay showed that all purified forms of Shh are cholesterol-modified, with a variable percentage of dually lipidated protein as if palmitoylation was subsequent to cholesterol modification [4]. On the other hand, an uncleavable as well as a mutated form of Shh, both lacking the cholesterol modification, have been found to carry [3H]-palmitic acid or ${ }^{125}$ I-iodo palmitate when metabolically labeled in cultured cells $[13,16]$. Similarly, a non-cholesterol-modified Drosophila Hh transgene displayed in vivo activity that depends on its palmitate, suggesting that cholesterol addition of $\mathrm{Hh}$ was not a prerequisite for palmitoylation [11].

Finally, one could speculate that acylation affects the routing of Hh secretion, as it was recently demonstrated that acylation of a similar morphogen, Wnt-3a protein, with a palmitoleic acid is required for appropriate trafficking from the ER [17]. However, in vivo analysis of a form of Hh lacking only the palmitic acid modification showed that it could reach and activate target cells as if $\mathrm{Hh}$ secretion was independent of palmitoylation [5]. Nevertheless, one must take these studies with caution, as they are hampered by the fact that this variant was expressed at a nonphysiological level possibly causing misrouting within producing cells.

How the activity of the acyl-transferase Skn is regulated is also unclear, although another mammalian member of the MBOAT familly, Gup1, has been shown to negatively regulate the N-terminal palmitoylation of Shh, very likely by competing with Skn rather than catalyzing depalmitoylation [14]. This is consistent with biochemical studies showing that Shh palmitoylation does not follow cycles of palmitoylation-depalmitoylation and is thus not reversible [16]. In addition, palmitoylation or other N-terminal hydrophobic modifications of Shh greatly increase its activity in a cell-based assay that does not require secretion or transport without affecting its ability to bind Patched (Ptc) receptor [18]. This suggests that the possible role of palmitoylation is to modulate Hh activity by increasing its stability or by changing its affinity for associated cofactors.

\section{Secretion and Release of Lipid-Modified Hh}

During embryonic development, Hh activity can exert an effect over large fields of cells, for example up to $50 \mu \mathrm{m}$ in Drosophila wing imaginal disc and $300 \mu \mathrm{m}$ in vertebrate neural tube or limb bud [2]. The long-range activity of Hh raises the question of how a dually lipidated protein can escape the membranes of producing cells to directly activate cells distant from its source of production.

Earlier studies have shown that the cholesterol moiety attached to Hh plays an important role in the protein's membrane retention as it causes strong membrane binding (reviewed in [3]). Indeed, in vitro engineered forms of Hh lacking cholesterol have been found to freely dissociate from cells after secretion, indicating that the cholesterol adduct can function as an anchor that restricts the mobility 
of the signal. Then how is cholesterol-modified Hh released from the lipid bilayer? A biophysical study revealed that cholesterol alone anchors proteins to membranes with significant strength and revealed a spontaneous desorption half-time of several hours [19], suggesting that a specific cellular activity or machinery is necessary for Hh release in a shorter time lapse.

Hh may be released from specialized membranes as lipidation of Hh allows its association with sterol-rich membrane microdomains, such as lipid rafts in Drosophila and in mammalian cells [13, 20]. Interestingly, optical imaging using tagged forms of Hh in Drosophila identified nanoscale oligomers at the cell surface [21]. Moreover, a higher order of Hh clustering was also observed and shown to involve ligand interactions with heparan sulfate proteoglycans (HSPG, see below), resulting in visible clusters of the Hh ligand at the cell surface. Mutants of Hh that are impaired in oligomerization lose their long-range activity, suggesting that nanoscale organization of $\mathrm{Hh}$ at the plasma membrane is essential for Hh to move a distance [21] (see below).

Why is this so? Within membrane microdomains, Hh may encounter proteins important for its release and efficient spreading. For example, it appears that packaging of $\mathrm{Hh}$, destined for long-range movement, partially requires the cytoplasmic membrane-scaffolding proteins Reggie1/flotillin2 [22], typical members of lipid rafts [23]. Interestingly, overexpression of Reggie1 in the wing imaginal disc of Drosophila increased the level of extracellular Hh and its spreading, as well as the activation of the pathway at long range [22]. Reduction of Reggie in the wing disc causes a weak reduction of long-range Hh targets, without affecting shortrange signaling [22], indicating that diverse routes may be taken by different pools of Hh (i.e. long vs. short range). In fact, several other proteins have been shown to be specifically required for long-range, but not short-range, bound Hh. This is discussed below.

Some insight into the process of $\mathrm{Hh}$ release from producing cells came from the identification of the dispatched (disp) gene that is predicted to encode a 12 transmembrane protein, containing a sterol-sensing domain (SSD), and is required in both Drosophila and mouse Hh-producing cells to transport the lipid-modified $\mathrm{Hh}$ protein [24, 25]. Importantly, autocrine activity of Shh in the notochord and juxtacrine Hh activity in imaginal discs were not affected in disp mutant animals, but no long-range signaling was observed [24, 26, 27]. These observations suggest that $\mathrm{Hh}$ secretion, release, and its long-range activity are closely linked to lipid modification and likely employ a novel intracellular machinery to secrete and release the membrane-anchored Hh protein.

While the exact function of Disp remains unknown, it has been suggested to act as a proton gradient-driven transporter of the Resistance-Nodulation-Division family, as mutations in conserved residues impair its activity [27]. These permeases use a proton electrochemical gradient to function as antiporters in driving out substrates such as hydrophobic drugs, heavy metals, and endogenous compounds. From this homology, it is not clear whether Disp acts by changing the membrane microenvironment around $\mathrm{Hh}$ favoring clustering or whether it promotes the release 
of lipid-modified proteins, as it has been shown for another transmembrane transporter of the bacterial ATP binding cassette family, the LolCDE complex from Escherichia coli [28].

It is intriguing that Disp contains a SSD, similar to the one present on proteins which are involved in cholesterol homeostasis or trafficking, including NiemannPick C1 (NPC1) [29]. Because only the cholesterol-modified form of $\mathrm{Hh}$ is sensitive to Disp activity, Disp-dependent Hh secretion could involve its SSD domain. This domain has been shown to be important for vesicular trafficking regulated by cholesterol level. Because association has been shown between the SSD domain of NPC1 and free cholesterol [30], it is possible that Hh secretion depends on the interaction between the SSD domain of Disp and the cholesterol moiety of Hh.

Based on these studies, and on the fact that in disp mutants, Hh protein production, and processing are not affected, several possible nonexclusive roles have been proposed: (1) Disp may be involved in the intracellular trafficking of $\mathrm{Hh}$ to the appropriate membrane microdomain dedicated to its secretion. Indeed, we have previously shown that in the absence of Disp function, cholesterol-modified $\mathrm{Hh}$ remains localized to the basolateral membrane of polarized embryonic epidermal secreting cells while it is apically localized in wild-type siblings [11]. However, no specific abnormal apical/basal localization of Hh was observed in disp mutant epithelial cells of the wing imaginal disc [24] (D'Angelo and Thérond unpublished observation); (2) $\mathrm{Hh}$ is also secreted under its monomeric form, however, its longrange activity was shown to be dependent on the clustering of $\mathrm{Hh}$ multimers with HSPG [21] and on the packaging into lipoprotein particles [31]. Interestingly, visible clusters of $\mathrm{Hh}$ are absent in disp mutant animals suggesting that it may control the oligomerization of $\mathrm{Hh}[11,32]$. It is thus possible that Disp directly promotes the clustering of $\mathrm{Hh}$ which is then sequentially associated with other proteins; and (3) Disp may facilitate a direct release of Hh from the plasma membrane into the extracellular space. However, secretion of an active form of Shh was observed from Disp null fibroblast, arguing against this model [25]. In summary, we believe that Disp functions in the formation of an active fraction composed of Hh oligomers and other proteins (HSPGs, lipoprotein particles, see below) dedicated to long-range transport.

\section{Hedgehog Spreading: A Dilemma in Motion}

After release, several modes of Hh movement have been suggested including (1) transcytosis (where proteins are passed cell-to-cell by alternating endo- and exocytosis events) [33], or (2) movement on various vehicles through the extracellular matrix (ECM). However, various studies in Drosophila have illustrated that blocking dynamin - the "motor" involved in many cell processes, including endoand exocytosis - has no effect on the spreading of $\mathrm{Hh}$ in either the wing disc $[34,35]$ or in the fly embryo [36]. Thus in flies, the first model has been generally rejected 
in favor of the second. This prediction is certainly bolstered by numerous studies which have found that secreted or membrane-attached proteins which make up the ECM can regulate the spreading or signaling of Hh. One group of these proteins attracting much interest is the HSPGs.

Proteoglycans are proteins which have long, unbranched glycosaminoglycan (GAG) chains (sugar polymers) attached to specific serine residues on their protein core. HSPGs have heparan sulfate (HS)-containing GAG chains. Originally viewed as important ECM components, recent evidence has highlighted the critical role of these sugar-modified proteins in morphogenesis and development. Accordingly, mis-regulation of the different proteins involved in HSPG biosynthesis manifests in a large variety of phenotypes [37].

A number of studies have suggested that HSPGs, more specifically the glypicans - a subtype of HSPGs anchored to the plasma membrane though a glycerophosphatidylinositol (GPI) motif - play an important role in the cells which secrete or spread Hh. Glypicans can accumulate in membrane microdomains and appear to be important for the recruitment of Hh into visible clusters (as discussed above) [21]. In addition, HSPGs at the plasma membrane may be involved in Hh stability, as loss of tout velu (ttv) or sister of tout velu (enzymes of the EXT family involved in the GAG chain polymerization) causes a noticeable reduction in Hh levels in the wing imaginal disc [38]. Indeed it has been demonstrated that glypicans recruit lipophorin (a Hh vehicle - see below) to Drosophila Hh secreting cells [39]. Thus, the GPI-anchored glypicans appear to be involved in Hh organization at the plasma membrane of secreting cells, Hh stability, and perhaps even Hh vehicle recruitment and loading.

Glypicans also have the ability to move within tissues. Eugster et al. [39] showed that glypicans are commonly shed by wing imaginal discs, moving into surrounding tissues where they are internalized. Furthermore, both fly glypicans Dally-like (Dlp) and Dally appeared to fractionate with $\mathrm{Hh}$ and lipoproteins in density-gradient experiments, through interactions with their GPI and the GAG chains [39], suggesting that they can move with these Hh carrying particles. Moreover, the fact that released glypicans, Hh, and lipoproteins are co-localized in endosomes in receiving cells indicates that they may be internalized together [39]. Altogether, fly glypicans do not only play a role in Hh secretion, but also appear to be associated and move with Hh carriers with which they are probably also internalized in receiving cells. This suggests that glypicans could also play an active role in the spreading of morphogens such as Hh.

\section{Extracellular Gradient Formation by HSPGs}

Control of $\mathrm{Hh}$ movement and regulation of extracellular $\mathrm{Hh}$ gradient formation by HSPGs is an attractive hypothesis and has been researched in various labs. Hh has a high affinity for HSPGs that reside at the cell surface [40]; however, Hh is unable 
to enter or cross a field of mutant cells which are deficient in HSPG assembly, as is observed in mutants for proteins which are involved in HSPG biosynthesis like ttv and sulfateless (a sulfotransferase involved in GAG chain modification) [34]. In these cases, $\mathrm{Hh}$ is found accumulated at the edge of the mutant tissue, proximal to the source, [41-44] and cannot spread to activate signaling within or past the mutant field [44]. Furthermore, the dependence of Hh movement toward HSPGs is specific to the cholesterol modification as contrary to fully modified Hh, noncholesterol or non-palmitoyled forms of $\mathrm{Hh}$ can spread through a field of cells deleted for $t t v$ activity. This is mimicked in cells where glypicans themselves (Dally and Dlp) are lost in the embryo, where Hh mobility is repressed [34]. Therefore, in addition to regulating $\mathrm{Hh}$ stability or membrane localization in secreting cells, glypicans are thought to be involved in an active process of Hh movement from one cell to the next, perhaps by passing the morphogen from GAG chain to GAG chain.

Recent evidence from flies has suggested that one glypican, in particular, may play an important role in Hh movement. In Drosophila, dally mutant wing imaginal discs show weakly decreased Hh signaling, and data indicate that Dally is involved in posterior Hh stability and recruitment of lipophorin [39]. Indeed, Dally itself appears to be present in high levels at the apical pole, and boosts Hh levels at the apical membrane of disc cells. Moreover, Dally release may aid in Hh secretion at this pole and long-range spreading through the ECM found in the apical lumen [45]. Certainly, overexpression of secreted-Dally ectopically activates Hh signaling far from the Hh source, something which is not seen when a transmembranetethered form of Dally is expressed [45, 46].

The protein Notum has been studied in both flies and mammals due to its HSPGrelated phenotypes. In flies, it has been suggested that Notum acts negatively on the Wnt pathway through its regulation of Dally [47, 48], and studies in mammalian cells have confirmed the ability of Notum to act as a PLC-like lipase and cleave GPI-anchor proteins, including various glypicans [49]. In flies, recent genetic studies have shown that Notum, like Dally, specifically regulates longrange signaling of $\mathrm{Hh}$. Indeed, Dally is unable to augment Hh movement in the absence of Notum, suggesting a link between Notum-dependent release of Dally and Hh spreading [45].

How Dally and Dally-like release promotes long-range Hh spreading is not clear. However, it is possible that Dally augments the release of Hh on specific vehicles such as lipophorin [39] or allows it to spread in a controlled manner without being lost within the surrounding lumenal space. It is also feasible that Dally GAG chains could protect $\mathrm{Hh}$ from protease-mediated degradation within the ECM, and along these lines the levels of sulfatation on HSPGs were shown to be an important factor in ADAM protease-mediated Hh cleavage [50] (see below). In conclusion, in Drosophila, HSPGs are required for the stable retention of $\mathrm{Hh}$ on the cell surface, which results in the restricted and controlled movement of Hh through the surface of the epithelium. Whether glypicans mediate similar processes in vertebrates is an interesting question for future research. 


\section{A Similar Spreading Mechanism from Flies to Mice?}

In vertebrate models, like in the fly, Hh ligands can act at long range [2]. Given that the requirement of dynamin for Hh spreading has not been tested in vertebrate systems, it remains to be seen whether Hh moves through the ECM in a similar manner as is seen in Drosophila epithelial cells. Indeed, recent findings from the Roelink lab, showing the mammalian protein Disp may be important in Hh receiving cells for continued spreading, has led to the speculation that Hh may be recycled in receiving cells to be released for transcytosis [51]. This would, thus, mean that Hh movement in these tissues could be subject to regulation by a different set of proteins to those in invertebrates. However, the fact that several HSPGs and members of the EXT family, as well as other ECM proteins, can still regulate the non-autonomous signaling of Shh suggests at least some role of ECM in this process.

The specific roles of Hh-HSPG interaction have been tested in mice carrying mutations within a sequence essential for Shh-proteoglycan interactions (the Cardin-Weintraub sequence) [52]. These mutant mice presented growth abnormalities of the CNS, but were free of the patterning abnormalities usually associated with Shh reduction. Intriguingly, it was found that interaction of Shh with proteoglycans is essential for its role in precursor proliferation in the external granule layer of the cerebellum, and illustrated that in this model proteoglycan-Shh interaction altered the cellular response to Shh, as a different profile of target genes and transcriptional activators (Gli) was detected in cells with and without this interaction [52]. Within the developing brain, the extracellular proteoglycans worked to localize Shh to the proliferating zone and, once there, they regulate the cellular response to Shh which favors the activation of a specific subset of proliferationactivating target genes. Thus, proteoglycans, such as the glypicans, appear to be important in controlling Hh signaling by reducing spreading of the ligand. This is at odds with a role in $\mathrm{Hh}$ movement, but indicates that at least some forms of $\mathrm{Hh}$ may move through the ECM in vertebrate systems, where they encounter ECM proteins such as glypicans.

Various additional results have indicated that glypicans can adopt several different roles within the Hh pathway. For example, null-mice for the vertebrate glypican GPC3 have increased Hh signaling [40, 53], as GPC3 competes with Ptc for the Hh ligand and thus inhibits signaling. It does this by inducing Hh internalization and degradation, thereby reducing its availability to Ptc [40]. Thus in tissues such as the bones, where Indian $\mathrm{Hh}$, a vertebrate member of the Hh family, stimulates the development of the endochondral skeleton, KO mice for GPC3 have increased Ihh levels and thus increased signaling, leading to bone overgrowth [54].

Consistent with the idea that Hh moves through the ECM, several other secreted glycoproteins have been implicated in extracellular Hh stability, and control the non-autonomous signaling of Hh. In Drosophila mutant animals for the ECM protein Shifted (Shf), most Hh target genes have reduced expression [55, 56]. Shf associates with both Hh and other ECM proteins, such as HSPGs, and its loss decreases Hh stability, and therefore, signaling $[55,56]$. Shf may also play a role in 
Hh movement as an Hh-GFP fusion protein does not appear to spread as far when Shf is lost, and although this could arguable be due to reduced stability, experiments where Hh levels have been restored to normal by Hh overexpression in a shf mutant background still show a reduced zone of target gene activation [55], indicating that both stability and movement are affected. Additionally, like many other pathway members, Shf action is specific only to cholesterol-modified Hh [55]. Akin to Shf, in Zebrafish, the vertebrate-specific Scube2 protein, an EGF and CUB domain protein, has been implicated in Hh specification of myogenic tissue [57, 58]. Scube has been described to have a cell non-autonomous requirement in the Hh receiving cells and may thus be involved in the formation of the Hh gradient, like its EGF-like domain containing fly cousin, Shf. In conclusion, several ECM proteins, whether secreted or plasma membrane tethered, control Hh stability and gradient formation in vertebrates, indicating ECM-mediated Hh movement as a common mechanism among different phyla.

\section{Hh Uses Multiple Carriers for Its Travels}

In flies and in vertebrates, Hh acts both at short and long range [1]. When discussing $\mathrm{Hh}$ carriers, one should remember that, depending on the range of its activity, $\mathrm{Hh}$ might associate with different carriers. Whatever the carrier, Hh needs to be able to pass through the hydrophilic environment of a tissue and its lipid moieties need to be shielded or cleaved. Several mechanisms would be capable of solubilizing hydrophobic Hh protein and evidence has been produced for some of them (reviewed in [59]). Also, recent data have led to the proposition of new and interesting mechanisms.

Could Hh simply be released from the cell surface by cleavage? This theory has been tested in Drosophila, in which two transgenic fly lines have been generated that either contain a transmembrane anchored form of $\mathrm{Hh}$, Hh-CD2, or a Hh-GPI that anchors Hh via a glycosyl-phosphatidylinositol attachment to the outer leaflead of the plasma membrane. Interestingly, absence of a long-range effect of those forms was concluded, strongly arguing that in Drosophila wing disc Hh is not simply released from the cell surface by cleavage. However, in mammals, the picture might be different, as Dierker et al. speculated that proteases, or lipases, could cleave and release Shh from membranes once it has been directed there. Indeed, they recently found that metalloproteases of the ADAM family function as the so-called "sheddases" in cells [50]. In particular, the action of secreted ADAM proteins, but not restricted to ADAM 17, leads to the removal of plasma membranebound $\mathrm{Hh}$ from mammalian Bosc23 cells. Interestingly, this group shows that an active form of Shh can be obtained from the medium that is devoid of both $\mathrm{N}$ - and C-terminal lipid modifications. Whether such mechanism also exists in intact tissues or in other species still needs to be validated.

For short-range transport, Hh could be handed over to the neighboring cell via direct cell-cell contact. ECM components such as HSPGs may be pivotal in this process. 
Dally and Dally-like, as discussed above, have been widely implicated in Hh binding, and given that cholesterol-modified Hh cannot cross a field of cells depleted of HSPGs, an involvement in lateral diffusion has been suggested in Drosophila [48]. Although cleaved Dally participates in Hh long-range spreading [45], it certainly remains possible that noncleaved proteoglycans also mediate short-range movement of Hh along the cell surface by transferring Hh from one HSPG on one cell to another one located next to it.

Compelling experimental evidence points to the existence of multiple Hh carriers for long-range transport of Hh. These might work in parallel and/or depend on the tissue type and developmental stage of the organism as well as on the source of Hh. Four different long-range carriers have been described:

1. Micelles formation by Hh (via self-aggregation or lipid binding proteins) has been proposed to shield the ligand's lipids attachments. Indeed, the presence of multimeric $\mathrm{Hh}$ complexes has been known for a long time. Closer analysis of these forms has revealed large soluble multimers of 5-6 molecules of Shh-N in conditioned media of vertebrate cells [60], and multimers of Hh-N (of $160 \mathrm{kDa}$ ) are also present in conditioned culture media from Hh-producing Drosophila Schneider cells $[13,32]$. What could aid the formation of these structures? It is thought that the hydrophobic cholesterol moiety plays an essential role, perhaps by favoring micelle-like structure with interactions between lipids forming a lipophilic interior. Intriguingly, in Drosophila, absence of palmitoylation does not prevent Hh multimer nor visible cluster formation, but Shh that lacks palmitate fails to multimerise [13]. Additionally, the formation of these palmitatemediated aggregates is physiologically important, as abrogation of higher molecular weight complexes leads to a loss of long-range signaling in vertebrates.

While the interaction of $\mathrm{Hh}$ molecules in these complexes is of a non-covalent nature, Dierker et al. additionally described covalently cross-linked Hh oligomers [50]. The formation of these so-called "undisruptable" complexes depends on transglutaminase and heparan sulfate activity [50], and whether this form exists throughout the Hh-producing species remains to be investigated.

2. Akin to micelle, Hh could be secreted as an integral component of lipoprotein particles. Lipoprotein particles seem to be involved in the transport of systemic Hh in Drosophila. They are made up of cholesterol, phospholipids, and scaffolding proteins - apolipoproteins, the latter two components building a monolayer membrane. It is conceivable that $\mathrm{Hh}$ is anchored within lipoprotein particles via its attached lipid and/or cholesterol. Lipoprotein particles are produced in the fat body (a tissue functionally related to the liver) of the fly and are secreted into the hemolymph in which they circulate and reach other tissues. The reduction of lipophorin levels in the fat body leads to a significant remote effect on $\mathrm{Hh}$ spreading and signaling in peripheral tissues [31]. Lipoprotein-like particles also exist in higher vertebrates, however, whether these particles have a physiological function in the transport of Hh protein remains to be investigated. 
3. A third possibility is that Hh could be directed to secretory multivesicular bodies (MVBs). External vesicles, the so-called exosomes, have recently been suggested as carriers for Hh-related peptides in Caenorhabditis elegans, although a direct prove has not yet been demonstrated [61]. Nevertheless, the membrane-bound V0 sector of the vacuolar $\mathrm{H}^{+}$-ATPase (V-ATPase), an integral membrane component of MVBs, acts along this pathway leading to the apical secretion of exosomes containing Hedgehog-related peptides [61]. It is an intriguing possibility that similar mechanisms exist in other organisms in order to release Hh. For instance, Shh has been observed in extracellular vesicular structures called nodal vesicular particles (NVPs) located at the surface of mouse ventral node during embryonic development [62]. It has been speculated that NVPs, which consist of a membrane sheath and a lipid core, enable transportation of Shh over vast distances without solubilization [62]. Further investigation into the nature and origin of these vehicles is merited.

4. Membrane-associated Hh may also provide another active form of the ligand. Although it is generally accepted that $\mathrm{Hh}$ is released in a soluble form, up to $90 \%$ is found in Drosophila in a membrane-containing fraction including the plasma membrane [31]. This cell surface associated Hh in producing cells could feasibly be extracted by adjacent receiving cells. The Hh receptor Ptc on those cells might internalize Hh which then results in Hh signaling pathway activation [63]. Interestingly, in Drosophila imaginal discs, long cellular protrusions, called cytonemes, are found to extend toward certain morphogen-expressing boundaries [64]. Although no direct evidence for their involvement in Hh signaling has been demonstrated, the morphogen Decapentaplectic (Dpp) is found in cytonemes through which it is directed to its receptor Thickveins in Dpp-responsive cells [65]. Whether Hh uses this mode of transport as a bridge to reach its receptors over several cell diameters is still elusive, but has recently become under intense scrutiny.

From these data, we can conclude that Hh is transmitted by a variety of diverse carriers, each of which may provide unique properties to Hh, allowing differential stability, signal duration, and/or range of activity, through dissimilar biophysical characteristics and composition of these carriers. However, one must reiterate that controversies still exist about how these numerous $\mathrm{Hh}$ forms exert a combined signaling activity on ligand-receiving cells, and that where and how these vectors are assembled also presents a "black-box" in our understanding. Inventive and highly technical investigations are needed to shed light on these intriguing questions.

While it has been well established that constitutive activation of the Hh pathway (by loss of function of the Hh receptor Ptc) is responsible for certain kind of cancers (medulloblastomas, rhabdomyosarcomas and basal cell carcinomas), the growth of other tumor types has been shown to be regulated by an autocrine or juxtacrine manner involving secretion of Hh from these tumors. More recently, a new paracrine model has been proposed in which tumor cells produce Hh that stimulates surrounding stromal cells to produce growth factors that in turn support tumor growth [66]. This model conforms much more to Hh developmental activities, 
which in most cases are typically paracrine [67]. In addition, the level of Hh and/or its carriers secreted from these tumor cells may correlate with their severity, as it has been shown in tumor biopsies from prostate cancer [68]. Thus, research focused on Hh secretion and spreading, and proteins involved in these processes is essential in the battle against Hh-related tumors.

\section{Conclusions}

While great effort is being made to understand and manipulate the signaling cascade triggered by the Hh ligand, less attention has directed toward understanding the role of aberrant amounts of secreted $\mathrm{Hh}$ might have on surrounding responsive tissue. This issue is of great importance as locally concentrated Hh triggers a strong and direct gene response. Although very little is known about (1) where within the producing cells $\mathrm{Hh}$ processing and the addition of its unique lipid modifications occur, (2) what intracellular route Hh takes to reach the plasma membrane, (3) what constitutes the cellular machinery that leads to secretion, and (4) what kind of vehicle Hh uses to exert particular activities, emerging evidence strongly suggests that these may occur in a very specific way. It is these unique features of this molecule that might provide the Achilles tendon for therapeutic targets in the fight for a wide variety of syndromes caused by abnormal endogenous secretion of Hh ligand.

\section{References}

1. Varjosalo M, Taipale J (2008) Hedgehog: functions and mechanisms. Genes Dev 22:2454-2472

2. Ashe HL, Briscoe J (2006) The interpretation of morphogen gradients. Development 133:385-394

3. Mann RK, Beachy PA (2004) Novel lipid modifications of secreted protein signals. Annu Rev Biochem 73:891-923

4. Pepinsky RB et al (1998) Identification of a palmitic acid-modified form of human Sonic hedgehog. J Biol Chem 273:14037-14045

5. Chamoun $\mathrm{Z}$ et al (2001) Skinny hedgehog, an acyltransferase required for palmitoylation and activity of the hedgehog signal. Science 293:2080-2084

6. Micchelli CA, The I, Selva E, Mogila V, Perrimon N (2002) Rasp, a putative transmembrane acyltransferase, is required for Hedgehog signaling. Development 129:843-851

7. Amanai K, Jiang J (2001) Distinct roles of Central missing and Dispatched in sending the Hedgehog signal. Development 128:5119-5127

8. Lee JD, Treisman JE (2001) Sightless has homology to transmembrane acyltransferases and is required to generate active Hedgehog protein. Curr Biol 11:1147-1152

9. Hofmann K (2000) A superfamily of membrane-bound O-acyltransferases with implications for wnt signaling. Trends Biochem Sci 25:111-112

10. Maity T, Fuse N, Beachy PA (2005) Molecular mechanisms of Sonic hedgehog mutant effects in holoprosencephaly. Proc Natl Acad Sci USA 102:17026-17031

11. Gallet A, Rodriguez R, Ruel L, Therond PP (2003) Cholesterol modification of hedgehog is required for trafficking and movement, revealing an asymmetric cellular response to hedgehog. Dev Cell 4:191-204 
12. Mitra K, Ubarretxena-Belandia I, Taguchi T, Warren G, Engelman DM (2004) Modulation of the bilayer thickness of exocytic pathway membranes by membrane proteins rather than cholesterol. Proc Natl Acad Sci USA 101:4083-4088

13. Chen MH, Li YJ, Kawakami T, Xu SM, Chuang PT (2004) Palmitoylation is required for the production of a soluble multimeric Hedgehog protein complex and long-range signaling in vertebrates. Genes Dev 18:641-659

14. Abe Y, Kita Y, Niikura T (2008) Mammalian Gup1, a homolog of Saccharomyces cerevisiae glycerol uptake/transporter 1, acts as a negative regulator for N-terminal palmitoylation of Sonic hedgehog. FEBS J 275:318-331

15. Miura GI, Treisman JE (2006) Lipid modification of secreted signaling proteins. Cell Cycle 5:1184-1188

16. Buglino JA, Resh MD (2008) Hhat is a palmitoylacyltransferase with specificity for N-palmitoylation of Sonic Hedgehog. J Biol Chem 283:22076-22088

17. Takada $\mathrm{R}$ et al (2006) Monounsaturated fatty acid modification of Wnt protein: its role in Wnt secretion. Dev Cell 11:791-801

18. Taylor FR et al (2001) Enhanced potency of human Sonic hedgehog by hydrophobic modification. Biochemistry 40:4359-4371

19. Peters C, Wolf A, Wagner M, Kuhlmann J, Waldmann H (2004) The cholesterol membrane anchor of the Hedgehog protein confers stable membrane association to lipid-modified proteins. Proc Natl Acad Sci USA 101:8531-8536

20. Rietveld A, Neutz S, Simons K, Eaton S (1999) Association of sterol- and glycosylphosphatidylinositol-linked proteins with Drosophila raft lipid microdomains. J Biol Chem 274:12049-12054

21. Vyas $\mathrm{N}$ et al (2008) Nanoscale organization of hedgehog is essential for long-range signaling. Cell 133:1214-1227

22. Katanaev VL et al (2008) Reggie-1/flotillin-2 promotes secretion of the long-range signalling forms of Wingless and Hedgehog in Drosophila. EMBO J 27:509-521

23. Langhorst MF, Reuter A, Stuermer CA (2005) Scaffolding microdomains and beyond: the function of reggie/flotillin proteins. Cell Mol Life Sci 62:2228-2240

24. Burke R et al (1999) Dispatched, a novel sterol-sensing domain protein dedicated to the release of cholesterol-modified hedgehog from signaling cells. Cell 99:803-815

25. Tian H, Jeong J, Harfe BD, Tabin CJ, McMahon AP (2005) Mouse Disp1 is required in sonic hedgehog-expressing cells for paracrine activity of the cholesterol-modified ligand. Development 132:133-142

26. Caspary $\mathrm{T}$ et al (2002) Mouse Dispatched homolog1 is required for long-range, but not juxtacrine Hh signaling. Curr Biol 12:1628-1632

27. Ma Y et al (2002) Hedgehog-mediated patterning of the mammalian embryo requires transporter-like function of dispatched. Cell 111:63-75

28. Yakushi T, Masuda K, Narita S, Matsuyama S, Tokuda H (2000) A new ABC transporter mediating the detachment of lipid-modified proteins from membranes. Nat Cell Biol 2:212-218

29. Kuwabara PE, Labouesse M (2002) The sterol-sensing domain: multiple families, a unique role? Trends Genet 18:193-201

30. Ohgami $\mathrm{N}$ et al (2004) Binding between the Niemann-Pick $\mathrm{C} 1$ protein and a photoactivatable cholesterol analog requires a functional sterol-sensing domain. Proc Natl Acad Sci USA 101:12473-12478

31. Panakova D, Sprong H, Marois E, Thiele C, Eaton S (2005) Lipoprotein particles are required for Hedgehog and Wingless signalling. Nature 435:58-65

32. Gallet A, Ruel L, Staccini-Lavenant L, Therond PP (2006) Cholesterol modification is necessary for controlled planar long-range activity of Hedgehog in Drosophila epithelia. Development 133:407-418

33. Vincent JP, Dubois L (2002) Morphogen transport along epithelia, an integrated trafficking problem. Dev Cell 3:615-623 
34. Han C, Belenkaya TY, Wang B, Lin X (2004) Drosophila glypicans control the cell-to-cell movement of Hedgehog by a dynamin-independent process. Development 131:601-611

35. Torroja C, Gorfinkiel N, Guerrero I (2004) Patched controls the Hedgehog gradient by endocytosis in a dynamin-dependent manner, but this internalization does not play a major role in signal transduction. Development 131:2395-2408

36. Gallet A, Therond PP (2005) Temporal modulation of the Hedgehog morphogen gradient by a patched-dependent targeting to lysosomal compartment. Dev Biol 277:51-62

37. Bulow HE, Hobert O (2006) The molecular diversity of glycosaminoglycans shapes animal development. Annu Rev Cell Dev Biol 22:375-407

38. Bornemann DJ, Duncan JE, Staatz W, Selleck S, Warrior R (2004) Abrogation of heparan sulfate synthesis in Drosophila disrupts the Wingless, Hedgehog and Decapentaplegic signaling pathways. Development 131:1927-1938

39. Eugster C, Panakova D, Mahmoud A, Eaton S (2007) Lipoprotein-heparan sulfate interactions in the Hh pathway. Dev Cell 13:57-71

40. Capurro MI et al (2008) Glypican-3 inhibits Hedgehog signaling during development by competing with patched for Hedgehog binding. Dev Cell 14:700-711

41. Bellaiche Y, The I, Perrimon N (1998) Tout-velu is a Drosophila homologue of the putative tumour suppressor EXT-1 and is needed for Hh diffusion. Nature 394:85-88

42. The I, Bellaiche Y, Perrimon N (1999) Hedgehog movement is regulated through tout veludependent synthesis of a heparan sulfate proteoglycan. Mol Cell 4:633-639

43. Takei Y, Ozawa Y, Sato M, Watanabe A, Tabata T (2004) Three Drosophila EXT genes shape morphogen gradients through synthesis of heparan sulfate proteoglycans. Development 131:73-82

44. Han C et al (2004) Distinct and collaborative roles of Drosophila EXT family proteins in morphogen signalling and gradient formation. Development 131:1563-1575

45. Ayers KL, Gallet A, Staccini-Lavenant L, Therond PP (2010) The long-range activity of Hedgehog is regulated in the apical extracellular space by the glypican Dally and the hydrolase Notum. Dev Cell 18:605-620

46. Takeo S, Akiyama T, Firkus C, Aigaki T, Nakato H (2005) Expression of a secreted form of Dally, a Drosophila glypican, induces overgrowth phenotype by affecting action range of Hedgehog. Dev Biol 284:204-218

47. Giraldez AJ, Copley RR, Cohen SM (2002) HSPG modification by the secreted enzyme Notum shapes the Wingless morphogen gradient. Dev Cell 2:667-676

48. Han C, Yan D, Belenkaya TY, Lin X (2005) Drosophila glypicans Dally and Dally-like shape the extracellular Wingless morphogen gradient in the wing disc. Development 132:667-679

49. Traister A, Shi W, Filmus J (2008) Mammalian Notum induces the release of glypicans and other GPI-anchored proteins from the cell surface. Biochem J 410:503-511

50. Dierker T, Dreier R, Migone M, Hamer S, Grobe K (2009) Heparan sulfate and transglutaminase activity are required for the formation of covalently cross-linked hedgehog oligomers. J Biol Chem 284:32562-32571

51. Etheridge LA, Crawford TQ, Zhang S, Roelink H (2010) Evidence for a role of vertebrate Disp1 in long-range Shh signaling. Development 137:133-140

52. Chan JA et al (2009) Proteoglycan interactions with Sonic Hedgehog specify mitogenic responses. Nat Neurosci 12:409-417

53. Song HH, Shi W, Xiang YY, Filmus J (2005) The loss of glypican-3 induces alterations in Wnt signaling. J Biol Chem 280:2116-2125

54. Capurro MI, Li F, Filmus J (2009) Overgrowth of a mouse model of Simpson-Golabi-Behmel syndrome is partly mediated by Indian hedgehog. EMBO Rep 10:901-907

55. Glise B et al (2005) Shifted, the Drosophila ortholog of Wnt inhibitory factor-1, controls the distribution and movement of Hedgehog. Dev Cell 8:255-266

56. Gorfinkiel N, Sierra J, Callejo A, Ibanez C, Guerrero I (2005) The Drosophila ortholog of the human Wnt inhibitor factor Shifted controls the diffusion of lipid-modified Hedgehog. Dev Cell 8:241-253 
57. Woods IG, Talbot WS (2005) The you gene encodes an EGF-CUB protein essential for Hedgehog signaling in zebrafish. PLoS Biol 3:e66

58. Kawakami A et al (2005) The zebrafish-secreted matrix protein you/scube2 is implicated in long-range regulation of hedgehog signaling. Curr Biol 15:480-488

59. Eaton S (2006) Release and trafficking of lipid-linked morphogens. Curr Opin Genet Dev $16: 17-22$

60. Zeng X et al (2001) A freely diffusible form of Sonic hedgehog mediates long-range signalling. Nature 411:716-720

61. Liegeois S, Benedetto A, Garnier JM, Schwab Y, Labouesse M (2006) The V0-ATPase mediates apical secretion of exosomes containing Hedgehog-related proteins in Caenorhabditis elegans. J Cell Biol 173:949-961

62. Tanaka Y, Okada Y, Hirokawa N (2005) FGF-induced vesicular release of Sonic hedgehog and retinoic acid in leftward nodal flow is critical for left-right determination. Nature 435:172-177

63. Incardona JP et al (2000) Receptor-mediated endocytosis of soluble and membrane-tethered Sonic hedgehog by Patched-1. Proc Natl Acad Sci USA 97:12044-12049

64. Ramirez-Weber FA, Kornberg TB (1999) Cytonemes: cellular processes that project to the principal signaling center in Drosophila imaginal discs. Cell 97:599-607

65. Hsiung F, Ramirez-Weber FA, Iwaki DD, Kornberg TB (2005) Dependence of Drosophila wing imaginal disc cytonemes on Decapentaplegic. Nature 437:560-563

66. Yauch RL et al (2008) A paracrine requirement for hedgehog signalling in cancer. Nature 455:406-410

67. Ingham PW, McMahon AP (2001) Hedgehog signaling in animal development: paradigms and principles. Genes Dev 15:3059-3087

68. Karhadkar SS et al (2004) Hedgehog signalling in prostate regeneration, neoplasia and metastasis. Nature 431:707-712 
黛 Springer

http://www.springer.com/978-1-4419-8434-0

Hedgehog signaling activation in human cancer and its clinical implications

(Ed.) J. Xie

2011, IX, 217 p. 24 illus., 10 in color., Hardcover

ISBN: $978-1-4419-8434-0$ 